\title{
Erratum to: Bilateral serous detachment of retina: an unusual mode of revelation of thrombotic thrombocytopenic purpura of favorable outcome with plasma exchange
}

\author{
Cherif Titah • Noémie Abisror • Aude Affortit • \\ Paul Coppo
}

Published online: 21 November 2013

(C) Springer-Verlag Berlin Heidelberg 2013

\section{Erratum to: Graefes Arch Clin Exp Ophthalmol}

DOI 10.1007/s00417-013-2306-7

Reason: Main authors are listed incorrectly.

\section{Corrected version:}

The author list should read as follows: Cherif Titah, Noémie Abisror, Aude Affortit, Paul Coppo

Marc Buffet needs to be removed from the author list.

The online version of the original article can be found under doi:10.1007/ s00417-013-2306-7.

\section{Titah $\cdot$ A. Affortit}

Fondation Adolphe de Rothschild, Service du Professeur

Cochereau, 75019 Paris, France

N. Abisror · P. Coppo

Centre de Référence des Microangiopathies Thrombotiques,

Département d'Hématologie, Hôpital Saint-Antoine, AP-HP,

et UPMC Univ Paris 06, Paris, France

\section{P. Coppo}

Unité Inserm 1009, Institut Gustave Roussy, 94805 Villejuif,

France

P. Coppo $(\square)$

Centre de Référence des Microangiopathies Thrombotiques,

Service d'Hématologie, Hôpital Saint-Antoine, Université Pierre

et Marie Curie, 184 rue du Faubourg Saint-Antoine, Assistance,

Publique - Hôpitaux de Paris,

75012 Paris, France

e-mail: paul.coppo@sat.aphp.fr 